\title{
Needle Biopsy of Recurrent Adenocarcinoma of the Prostate After Radical Prostatectomy
}

\author{
Mary G. Ripple, M.D., Steven R. Potter, M.D., Alan W. Partin, M.D., Ph.D., Jonathan I. Epstein, M.D. \\ Departments of Pathology (MGR, JIE) and Urology (SRP, AWP, JIE), The Johns Hopkins University School \\ of Medicine, The James Buchanan Brady Urological Institute, The Johns Hopkins Hospital, Baltimore, \\ Maryland
}

The objective of this study was to evaluate needle biopsy of recurrent prostate cancer after radical prostatectomy. We evaluated 37 cases of recurrent prostate cancer after radical prostatectomy that were diagnosed by needle biopsy between March 1984 and July 1998. Fifteen were from consultations in which contributors were uncertain of the diagnosis, and 22 were from men who had come to The Johns Hopkins Hospital for treatment. The median interval from radical prostatectomy to biopsy showing recurrent tumor was $\mathbf{4 0}$ months. There was no correlation between the interval to recurrence and either pathologic features of the biopsy and radical prostatectomy or various clinical features. The mean extent of adenocarcinoma in the biopsies was $3.2 \mathrm{~mm}$ (range, 0.1 to $18 \mathrm{~mm}$; median, $2 \mathrm{~mm}$ ). The length of recurrent cancer on biopsy correlated with an abnormal rectal examination $(P=.001)$. The mean Gleason score for the recurrent tumors was 6.5, which correlated with the grade of the radical prostatectomy cancer $(P=.005)$. The cancers often lacked overt histologic features of malignancy. Benign prostatic acini were seen in five cases $(14 \%)$, usually separate from the cancer. In 5 (33\%) of the consultation cases, we would not have been able to diagnose cancer if not for the fact that atypical prostate glands should not be present after radical prostatectomy. In well-sampled radical prostatectomies, margins were almost always positive, as was extraprostatic extension. In cases with less sampling, there was a higher incidence of organ-confined disease and margin-negative disease implying suboptimal processing of the radical prostatectomy. After radical prostatectomy, recurrent cancer on needle biopsies may be focal and difficult to diagnose and

Copyright () 2000 by The United States and Canadian Academy of Pathology, Inc.

VOL. 13, NO. 5, P. 521, 2000 Printed in the U.S.A.

Date of acceptance: November 12, 1999.

Funded by grants from NCI SPORE CA58236.

Address reprint requests to: Jonathan I. Epstein, M.D., Department of Pathology, The Johns Hopkins Hospital, 600 N. Wolfe Street, Baltimore, MD 21287; e-mail: jepstein@welchlink.welch.jhu.edu; fax: 410-955-0115. must be assessed differently than in patients who have not had surgery.

KEY WORDS: Adenocarcinoma, Local recurrence, Pathology, Prostatic neoplasms.

Mod Pathol 2000;13(5):521-527

Patterns of failure after radical prostatectomy for adenocarcinoma of the prostate include local recurrence, distant metastases, and a rising postoperative serum prostate-specific antigen (PSA) level with no evidence of local or distant failure. Most studies have concentrated on men who had biochemical evidence of progression as the sole indication of failure (1). The few reports that evaluated local recurrence concentrated on clinical issues, including (1) the use of radiotherapy to treat local recurrence (2); (2) the need for several biopsies to document local recurrence (3-5); (3) the use of ultrasound, serum PSA, and digital rectal examination to detect local recurrence (3-7); and (4) the sites of local recurrence, including the anastomotic site $(66 \%)$, bladder neck (16\%), retrovesical space (13\%), and unknown (5\%) (3).

Several studies also commented on the pathologic stage of the initial cancer at radical prostatectomy $(3,4,6-9)$. Only a couple of articles have provided any details on the histologic findings of locally recurrent cancer, with information limited to the Gleason score of the recurrent tumor $(3,7)$. The objectives of our study were to evaluate the histologic features of recurrent adenocarcinoma of the prostate after radical prostatectomy and to correlate these findings with reviewed clinical and radical prostatectomy data.

\section{MATERIALS AND METHODS}

The surgical pathology file of The Johns Hopkins Hospital Department of Pathology was searched from March 1984 to July 1998 for reports containing the word "prostate" combined with any of the other following terms: "recurrent," 
"bed," “fossa," “anastomosis," “anastomotic," "vesicourethral," and "peri-prostatic region." Clinical and pathologic material was reviewed to select cases that consisted of needle biopsies with recurrent prostate cancer after radical prostatectomy. Needle biopsies with recurrent adenocarcinoma of the prostate were reviewed for the following features: Gleason score; extent of adenocarcinoma; and the presence or absence of nuclear hyperchromasia, nuclear enlargement, cribriform glands, ductal features, crystalloids, blue intraluminal mucin, amphophilic cytoplasm, perineural invasion, and benign prostate glands. Prominent (readily visible) nucleoli were categorized as none, rare, moderate, and numerous. A subjective assessment was made as to whether the biopsy would have been diagnostic of cancer had there been no history of radical prostatectomy.

Thirty-seven cases of needle biopsy with recurrent prostate cancer after radical prostatectomy were evaluated. Radical prostatectomies were performed at The Johns Hopkins Hospital in 12 of $37(32 \%)$ of the cases and were performed at an outside hospital in the remaining 25 cases. Of the radical prostatectomies that were performed at the outside hospitals, the majority of the radical prostatectomy slides were reviewed in 7 of 25 (28\%) of those cases, a minority of slides were reviewed in 4 of 25 (16\%) of those cases, and only the surgical pathology report was reviewed in 14 of $25(56 \%)$ of those cases. Radical prostatectomy data were reviewed with regard to the Gleason score, extraprostatic extension, surgical margins, and seminal vesicle and lymph node involvement.

Clinical information was obtained from patient records for patients who were treated at The Johns Hopkins Hospital and via telephone interviews with urologists for cases that were diagnosed elsewhere. Subsequently, outside institutions were contacted for pathology reports and slides. Clinical data obtained included interval to recurrence, serum PSA at recurrence, digital rectal examination results, transrectal ultrasound examination findings, and the presence of metastatic disease at the time of local recurrence.

Statistical analysis was performed using a statistics graphic data measurement software program (STATA, College Station, TX). The two-sample $t$ test, rank sum test, and $\chi^{2}$ analysis were used to determine whether there were differences between consultation cases and patients who were treated at The Johns Hopkins Hospital. Correlation between clinical and pathologic findings was evaluated by regression analysis.

\section{RESULTS}

\section{Clinical Data}

Patients ranged in age from 53 to 75 years with a mean age of 65. Data on interval to first recurrence, as determined by the date of the initial documented elevation of PSA, were available for 35 of 37 patients (95\%). The PSA level at initial elevation was available for 31 of 37 patients (84\%); for 4 patients, information was present on when the first PSA elevation occurred but not the actual PSA level. The median interval from radical prostatectomy to the first biochemical evidence of recurrence was 16 months (range, 0 to 108 months); the postoperative serum PSA level never fell to zero in 2 of 37 patients (5\%). The median interval from radical prostatectomy to biopsy of local recurrence was 40 months (range, 3.5 to 132 months). The median serum PSA at time of the first biochemical failure was 0.6 $\mathrm{ng} / \mathrm{mL}$ (The Johns Hopkins Hospital definition of postoperative biochemical failure is $>0.2 \mathrm{ng} / \mathrm{mL}$ ), whereas the median serum PSA level at time of biopsy recurrence was $2.0 \mathrm{ng} / \mathrm{mL}$. The digital rectal examination was described as normal in 12 patients (41\%), indurated in 6 patients $(21 \%)$, and revealing a mass in 11 of the 29 patients (38\%) for whom these data were available. Transrectal ultrasound was suspicious for recurrent tumor in 10 of 22 patients (45\%) whose information was available. Only 3 of 29 patients (10\%) whose information was available had evidence of distant metastases, as evidenced by positive bone scans, at the time of local recurrence.

\section{Recurrent Cancer Biopsy Findings}

The Gleason scores of the 34 recurrent acinar cancers were as follows: $4(n=1) ; 5$ to $6(n=20)$; 7 $(n=10)$; and 8 to $10(n=3)$ (Fig. 1$)$. The average Gleason score was 6.5 . Three biopsies showed only ductal differentiation (8\%) and were not assigned a Gleason score (Fig. 2).

Prominent nucleoli were rare or absent in 14 patients (38\%) (Table 1, Fig. 3). Other histologic findings are depicted in Table 1 (Fig. 4). There were no differences in histologic features, with the exception of tumor extent (see below), between the cases that were consultations and those that were from patients who were treated at The Johns Hopkins Hospital. Cribriform glands were present in 12 patients $(32 \%) ; 2$ patients $(5 \%)$ had cribriforming glands only, and $5(14 \%)$ had more cribriforming glands than individual acini (Fig. 5). Benign prostatic acini were present in 5 patients (14\%), in most cases separate from the recurrent adenocarcinoma (Fig. 6).

In 5 of the consultation cases (33\%), we would not have been able to diagnose cancer if not for the 


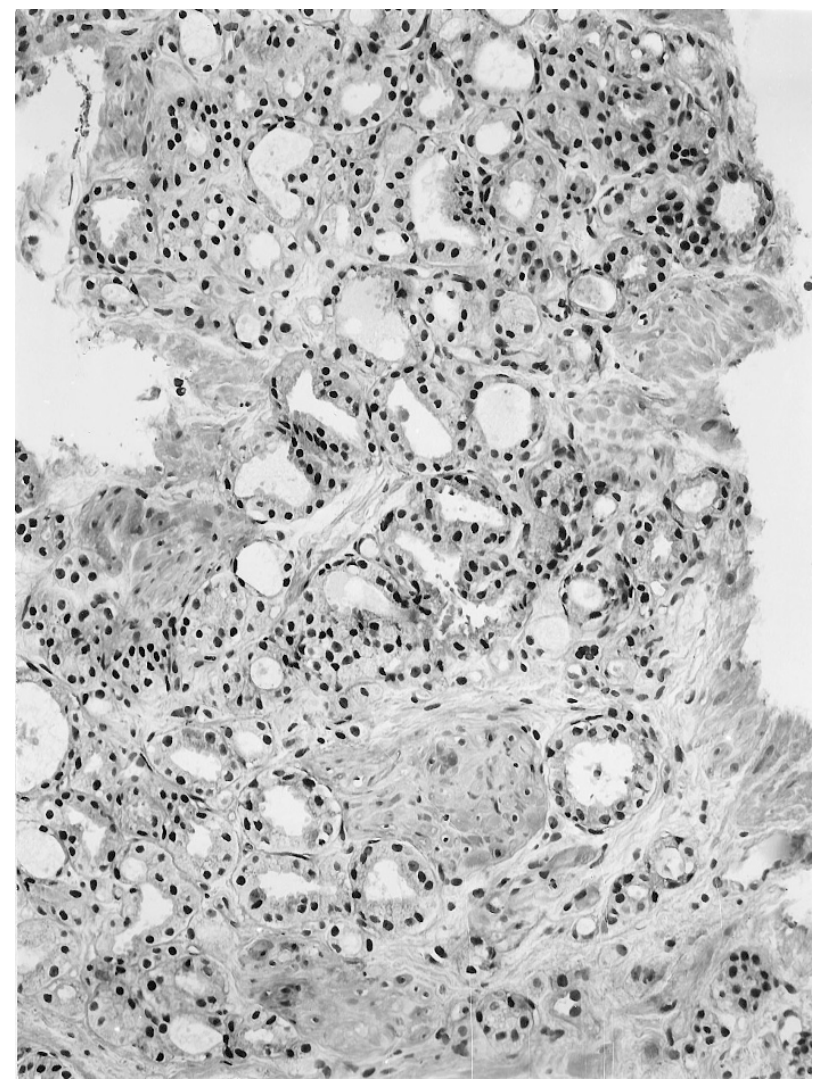

FIGURE 1. Recurrent adenocarcinoma of the prostate composed of individual crowded glands infiltrating smooth muscle bundles, consistent with Gleason pattern 3 tumor.

fact that atypical prostate glands should not be present after radical prostatectomy (Fig. 7). Ancillary PSA or prostate-specific acid phosphatase immunostains were used in 5 cases (14\%), but in none of the cases were the immunostains essential for the diagnosis.

The majority of the recurrences were in fibrous and smooth muscle tissue; only 4 cases of recurrence in skeletal muscle were $(10 \%)$ identified. The mean extent of adenocarcinoma in the biopsies was $3.2 \mathrm{~mm}$ (range, 0.1 to $18 \mathrm{~mm}$; median, $2 \mathrm{~mm}$ ). The extent of the adenocarcinoma was smaller in the true consultation cases with a mean of $1.7 \mathrm{~mm}$, as compared with $4.3 \mathrm{~mm}$ ( $t$ test, $P=.04$; rank sum test $P=.01$ ) for patients who were treated at The Johns Hopkins Hospital.

\section{Radical Prostatectomy Findings}

In 34 of 35 cases, radical prostatectomy slides or reports were available for review. The Gleason score of the radical prostatectomy averaged 6.8. Of these 34 cases, 11 had a Gleason score of 5 to 6,19 had a Gleason score of 7, and 4 had a Gleason score of 8 to 9. The Gleason score of the recurrent cancer on biopsy correlated with the radical prostatectomy Gleason score $\left(\chi^{2}, P=.005\right)$. Four radical prosta-

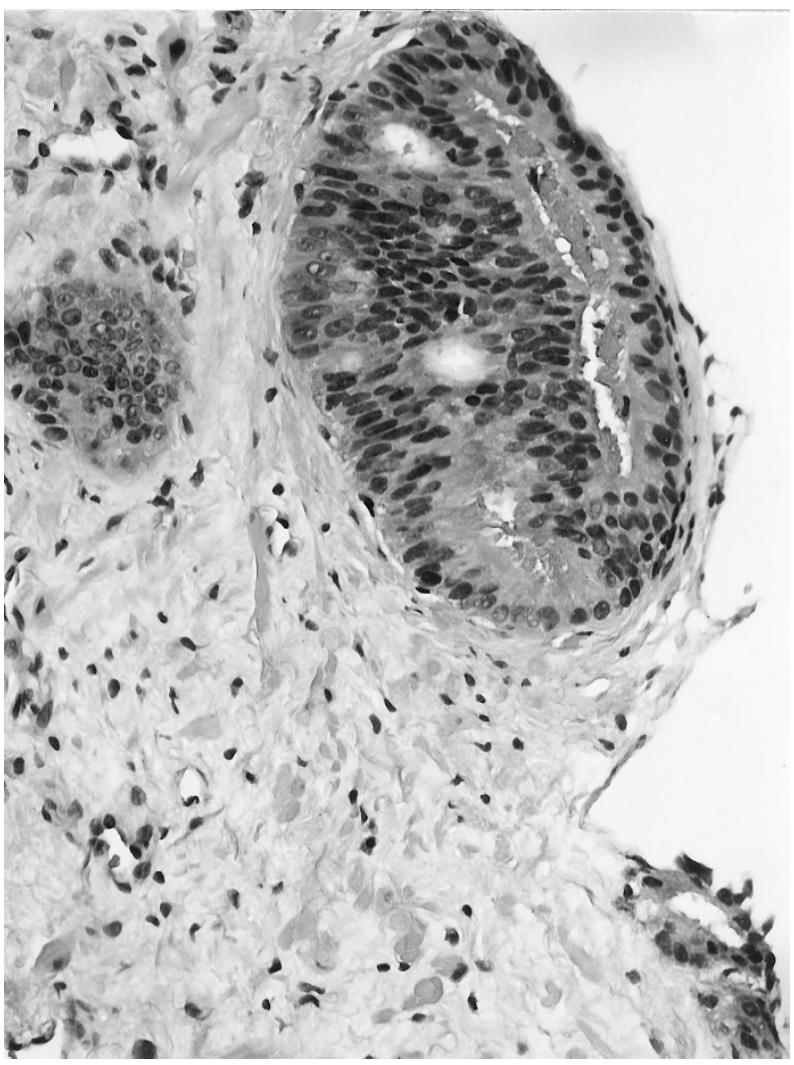

FIGURE 2. Recurrent adenocarcinoma with ductal features composed of a cribriform nest of tumor with tall columnar cells arranged in slit-like spaces.

TABLE 1. Histologic Findings of Locally Recurrent Cancer on Biopsy

\begin{tabular}{lc}
\hline Histologic Features & $\begin{array}{c}\text { No. of Cases } \\
(\%)\end{array}$ \\
\hline Prominent nucleoli & $5(14)$ \\
None & $9(24)$ \\
Rare & $7(19)$ \\
Moderate & $16(43)$ \\
Numerous & $29(78)$ \\
Nuclear enlargement & $11(30)$ \\
Hyperchromasia & $8(21)^{a}$ \\
Perineural invasion & $5(14)$ \\
Amphophilic cytoplasm & $5(14)$ \\
Mitoses & $4(11)$ \\
Blue mucin & $1(3)$ \\
Crystalloids & $5(14)$ \\
Benign glands present & $3(8)$ \\
Ductal features & $12(32)$ \\
Cribriforming glands &
\end{tabular}

${ }^{a}$ One case with all of the cancer showing perineural invasion.

tectomy specimens had focal and two had predominantly ductal adenocarcinoma.

Five tumors (15\%) were organ confined, 5 (15\%) had focal extraprostatic extension, and 25 (71\%) had established extraprostatic extension. Seminal vesicles were positive in 6 of 35 of the patients (17\%); only 1 patient (3\%) had positive lymph nodes at the time of radical prostatectomy (in one case, information on seminal vesicle invasion status was provided, despite a lack of information on other pathologic findings). 


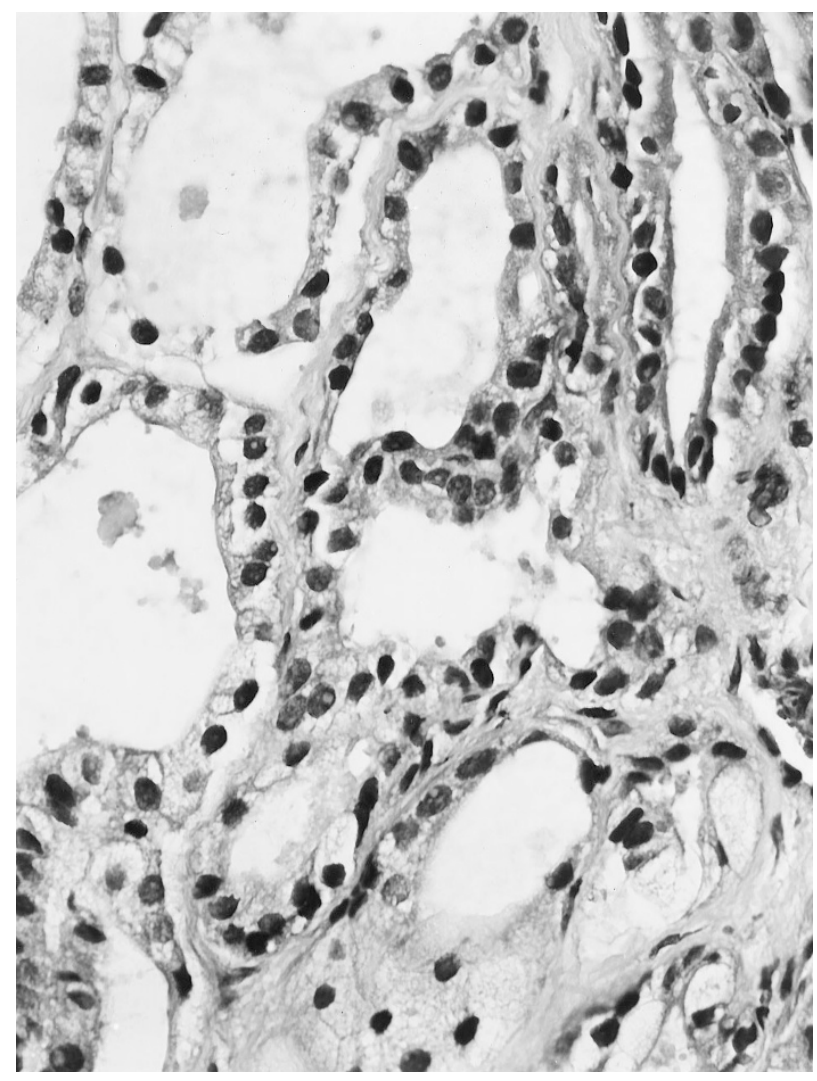

FIGURE 3. Recurrent adenocarcinoma with bland cytologic features.

Margins of resection were evaluated for all of The Johns Hopkins Hospital cases and for all outside cases in which radical prostatectomy slides were reviewed; however, 3 of 14 (21\%) of those cases in which only the radical prostatectomy report was available failed to mention margins of resection. Altogether, surgical margins were negative in 9 of 31 cases (29\%) with available information, equivocal in 3 of the cases (10\%), and positive in 19 (61\%). Extensive positive margins were noted in 3 of the 19 cases with positive margins. Of the 19 cases with positive margins, the apical region of the radical prostatectomy was involved in 13 of the cases (68\%). Only 1 of the 12 radical prostatectomies that were performed at The Johns Hopkins Hospital $(8 \%)$ had a negative margin. In that case, the prostate cancer was an incidental finding in a cystoprostatectomy specimen for bladder cancer, and the prostate was not sampled to the same extent (13 slides) as it would have been if it were a radical prostatectomy specimen. Of the outside cases, when the majority of the radical prostatectomy slides were reviewed, one of seven (14\%) had negative margins. This compares with 2 of 4 (50\%) with negative margins and 8 of $11(73 \%)$ with negative margins in cases with either a limited number of slides available for review or no slides reviewed, respectively.

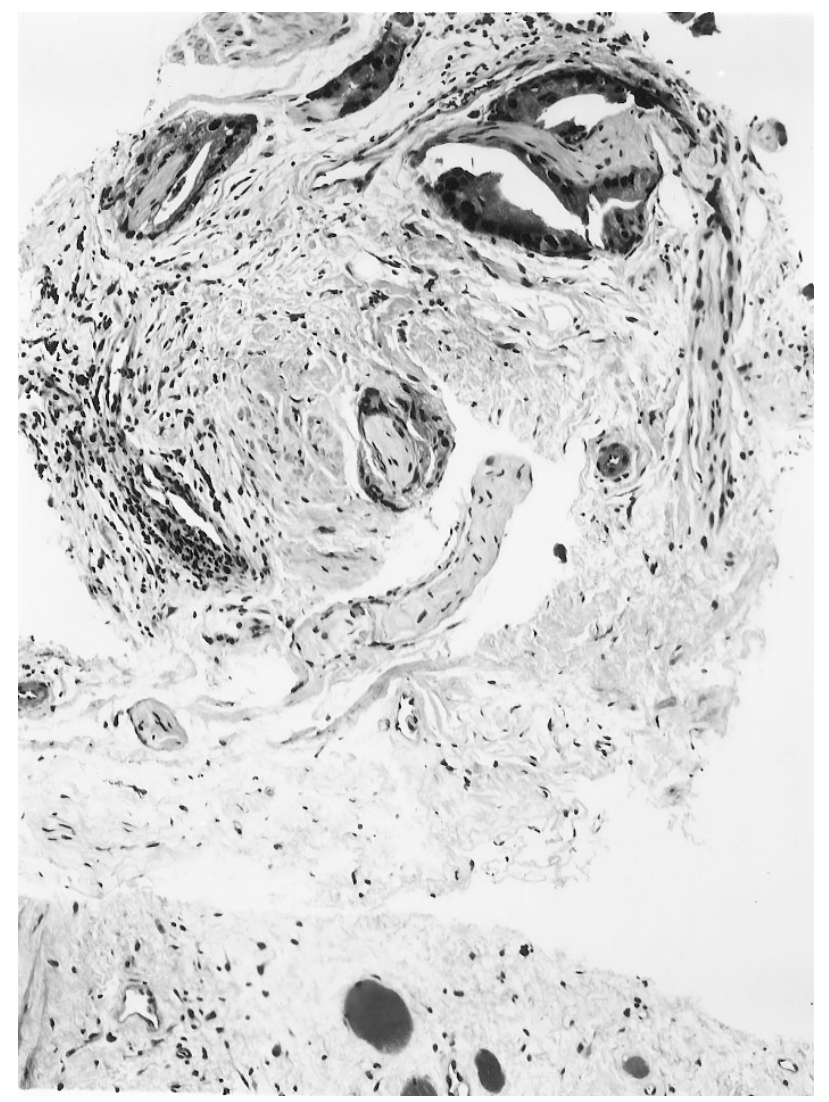

FIGURE 4. Recurrent adenocarcinoma solely composed of tumor encircling nerves. Tumor is seen infiltrating fibrous tissue and skeletal muscle (bottom).

\section{Correlation Between Clinical and Pathologic Findings}

More extensive recurrent cancer on biopsy correlated with a positive digital rectal examination ( $t$ test, $P=.001$ ). The following correlations reflect an analysis of all patients and a subset analysis of only those patients who underwent surgery at The Johns Hopkins Hospital. Higher serum PSA values at the time of the biopsy did not correlate with greater extent of adenocarcinoma at recurrence or a positive digital rectal examination at recurrence. There was no correlation between the interval to recurrence and Gleason score on the biopsy, Gleason score at radical prostatectomy, extent of adenocarcinoma in the biopsy, serum PSA at the time of the biopsy, extraprostatic extension, positive margins, or positive digital rectal examination at recurrence. A positive digital rectal examination at recurrence did not correlate with radical prostatectomy extraprostatic extension, positive margins, or Gleason score.

\section{DISCUSSION}

It has been estimated that after radical prostatectomy for clinical Stage T2A (palpable, unilateral) prostate cancer, 6 to $7 \%$ of tumors locally recur (1). 


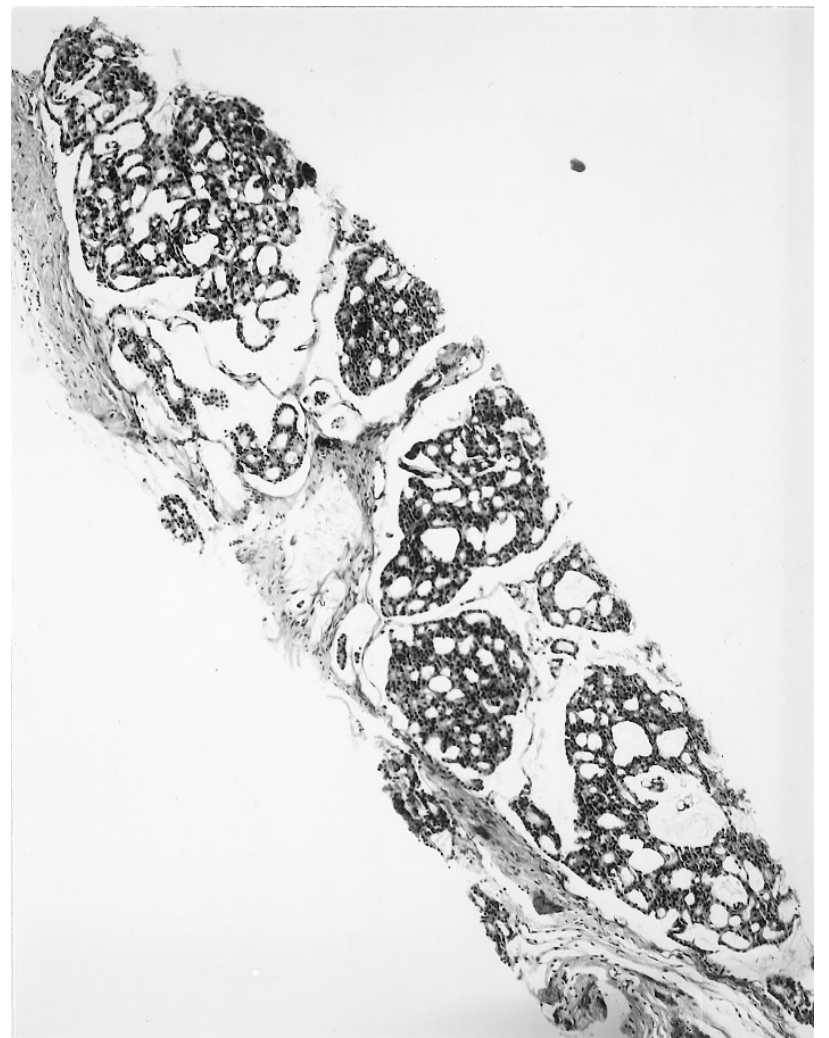

FIGURE 5. Recurrent cribriform adenocarcinoma

However, some of these studies have defined local recurrence as palpable induration at the site of surgery with an elevated serum PSA level and without radiographic evidence of distant metastases (1). Fewer studies have examined the issue of local recurrence documented by needle biopsy. One of the difficulties of studying this issue is that there are no uniform guidelines on when postoperative biopsies should be performed. Practices range from routine biopsies for men who have rising postoperative serum PSA levels to reliance on clinical findings to establish a diagnosis of recurrent prostate cancer. Because of this variance, the findings in this study may not be representative of all men who have recurrent prostate cancer. To account for some of the potential bias, we evaluated separately the patients who were sent in for consultation as opposed to those who came to our institution for therapy.

Several investigators have demonstrated the difficulty of diagnosing recurrent adenocarcinoma on biopsy, sometimes requiring the patient to have several needle biopsies over time (3-5). There are conflicting studies in the literature of whether transrectal ultrasound or digital rectal examination has greater sensitivity in detecting local recurrence (3-7). Our study agrees with those in the literature that found that, with the exception of an elevated postoperative serum PSA level, other clinical findings may not necessarily suggest a local recurrence.

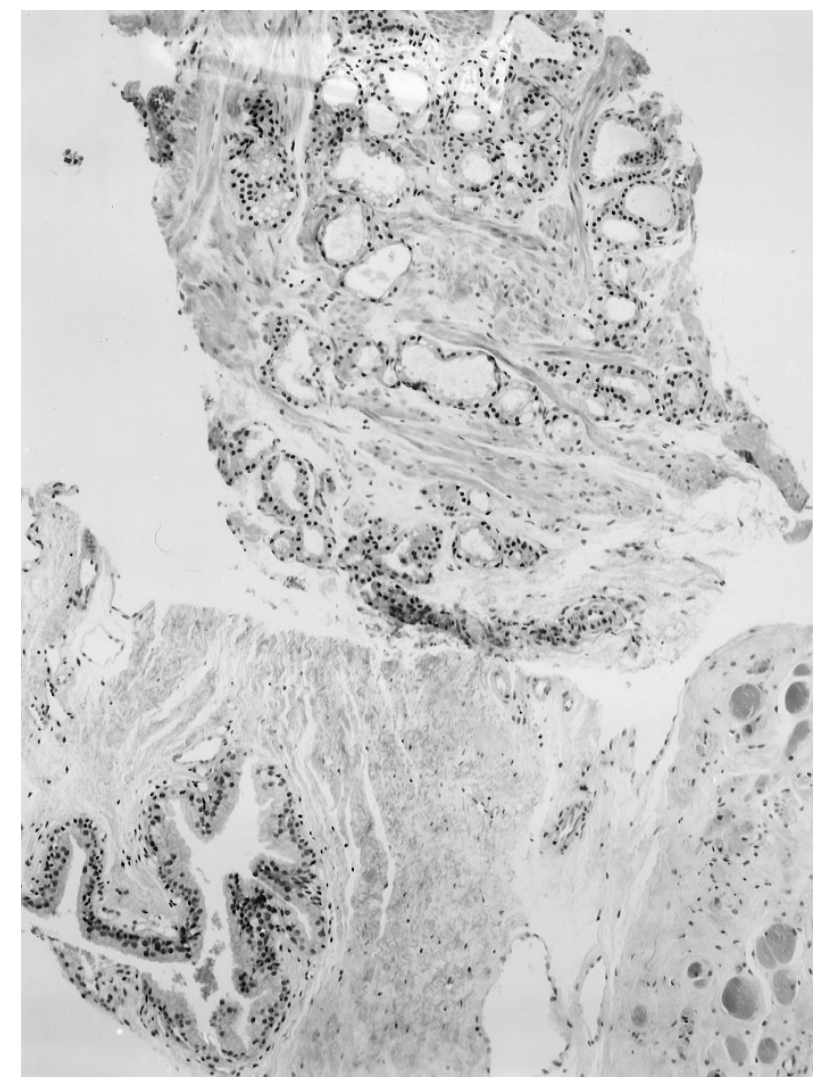

FIGURE 6. Recurrent adenocarcinoma (top) with benign prostate glands (bottom).

Within the literature correlating radical prostatectomy findings with local recurrence, some of the findings are expected, yet others are not. As expected, a high proportion of patients have advanced disease. The incidences of positive seminal vesicles and/or lymph node metastases range from 27 to $53 \%$ (Table 2) $(3,4,7,8)$. The reason for the somewhat lower incidence in our material $(20 \%)$ probably relates to patient selection and a tendency for patients who are coming for radical prostatectomy at The Johns Hopkins Hospital to have relatively early clinical stage disease. Radical prostatectomy Gleason scores for men who had local recurrence was on average 6.4 in one study and 7 in another $(3,7)$, comparable to our data. Although a couple of studies found no correlation between the Gleason score of the radical prostatectomy and recurrence, we did demonstrate such a relationship $(5,7)$. The lack of correlation reported in the literature may reflect inaccurate grading of the locally recurrent cancer on needle biopsy. Often, this tumor is limited and there is a tendency to undergrade limited adenocarcinoma of the prostate (10).

The surprising data relating to radical prostatectomy and subsequent local recurrence are in the incidences of organ-confined disease and positive margins. Within the literature, 33 to $48 \%$ of the radical prostatectomy tumors associated with 

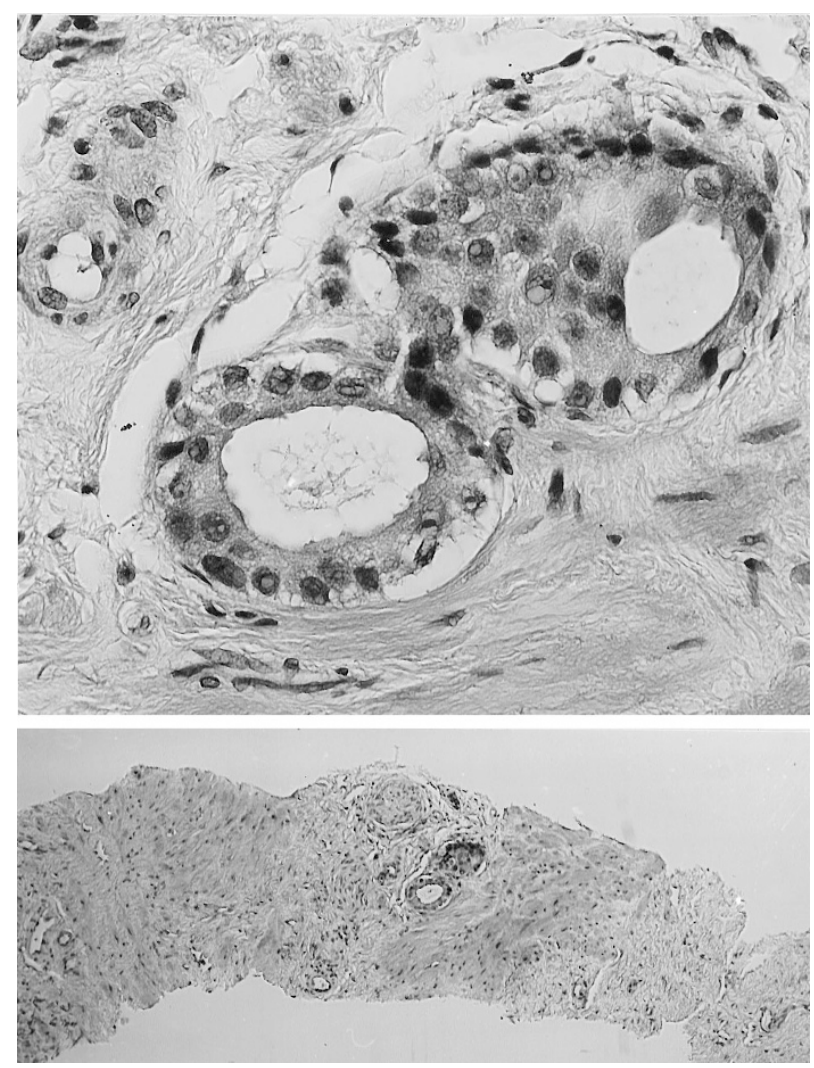

FIGURE 7. Minute focus of recurrent adenocarcinoma in fibrous tissue consisting of two atypical glands with prominent nucleoli. In part, this focus is diagnostic of cancer because the prostate has been removed and there are no other alternative explanations for finding atypical prostate glands in fibrous tissue.

biopsy-proven local recurrence were organ confined (Table 2). With the exception of the study by Connolly in which they stated that they stepsectioned 50 of 61 radical prostatectomy specimens, studies do not provide details on how the prostates were sampled. It is difficult to comprehend how organ-confined prostate cancers could locally recur unless one postulates spread via lymphatics to surrounding local soft tissue. Another alternative is intraoperative manipulation of the gland resulting in local tumor seeding. In our study, only $15 \%$ of the patients who had biopsy-proven local recurrence had organ-confined disease. The histologic recognition of extraprostatic extension may be difficult. Furthermore, extraprostatic exten- sion may be focal and present on only one or two histologic slides. In our data, $15 \%$ of our tumors showed focal extraprostatic extension, which could have been easily overlooked with limited sampling of the radical prostatectomy specimen. The relatively high proportion of patients who have biopsyproven local recurrence who have organ-confined disease is probably inaccurate and, in large part, reflects undersampling and underrecognition of extraprostatic extension.

The other unexpected finding reported in the literature relating to the correlation between radical prostatectomy findings and biopsy-proven local recurrence concerns margins of resection within the original resection specimen. One might anticipate that almost all radical prostatectomies associated with a subsequent local recurrence would have positive margins. However, in the literature, the incidence of positive margins ranges from 47 to $67 \%$ (Table 2). Only two studies stated that either all of the prostates were totally submitted or that in 50 of 61 cases the prostates were step-sectioned; these studies reported positive margin rates of $47 \%$ and $66 \%$, respectively $(3,9)$. Our data demonstrated a $61 \%$ positive margin rate. However, we found that in cases in which there was more sampling, there was a higher incidence of margin-positive disease. Consequently, many of the tumors reported as margin negative associated with a subsequent local recurrence may indeed have positive margins that were not recognized as a result of undersampling of the radical prostatectomy specimen.

It is also somewhat surprising that there was no relationship between findings at radical prostatectomy and the interval to recurrence. One might have expected that with more adverse findings in the radical prostatectomy there would have been a shorter time period to recurrence. This hypothesis was not supported by the findings in our study.

An additional finding in our study was the presence of benign prostatic glands in addition to the adenocarcinoma. Several studies have noted the presence of benign glands in biopsies after radical prostatectomy. Foster et al. (7) reported on eight patients who had benign glands on biopsy after radical prostatectomy. Of six patients who under-

TABLE 2. Locally Recurrent Prostate Cancer: Radical Prostatectomy Findings

\begin{tabular}{lcrc}
\hline \multicolumn{1}{c}{ Author (Reference) } & Organ Confined & $\begin{array}{c}\text { Positive Seminal } \\
\text { Vesicles/Lymph Nodes }\end{array}$ & Positive Margins \\
\hline Abi-Aad et al. (6) & $4 / 9(44 \%)$ & & $6 / 23(26 \%)$ \\
Fowler et al. $(4)$ & $11 / 23(48 \%)$ & $10 / 19(53 \%)$ & $9 / 19(47 \%)$ \\
Lange et al. (8) & $7 / 19(37 \%)$ & $4 / 15(27 \%)$ & $10 / 15(67 \%)$ \\
Van Den Ouden et al. (9) & $5 / 15(33 \%)$ & $27 / 61(44 \%)$ & $40 / 61(66 \%)$ \\
Foster et al. (7) & $10 / 61(16 \%)$ & $7 / 35(20 \%)$ & $19 / 31(61 \%)$ \\
Connolly et al. (3) & $5 / 34(15 \%)$ & $3 / 12(25 \%)$ & $11 / 12(92 \%)$ \\
Current study (all cases) & $1 / 12(8 \%)$ & \\
Current study (JHH radicals) & & & \\
\hline
\end{tabular}


went repeat biopsies, four were eventually shown to have, in addition, recurrent adenocarcinoma of the prostate. Fowler et al. (4) described six patients who had benign prostate glands on biopsy after radical prostatectomy. The only patient who underwent repeat biopsy was also found to have carcinoma. In the current study, we did not analyze cases in which there were only benign glands in the biopsy of the prostatic bed after radical prostatectomy. Benign glands on biopsy after radical prostatectomy imply that the prostate was not removed in its entirety. It remains unknown whether and how frequently the presence of only benign prostate glands left after radical prostatectomy can give rise to an elevated postoperative serum PSA level and the false impression of recurrent prostate cancer.

The current study demonstrated that recurrent cancer on needle biopsy may be focal and difficult to diagnose. This difficulty is reflected in cases that were sent in for consultation in which the extent of adenocarcinoma in the biopsies was often limited. Another factor that led to diagnostic difficulties was that the usual clues for the diagnosis of prostate cancer often were not present. We believe that there should be a lower histologic threshold for diagnosing recurrent prostate cancer in men who have had a radical prostatectomy. First, these men have a history of prostate cancer, in which rare malignant-appearing glands may be consistent with recurrent cancer yet insufficient to establish a primary diagnosis. This situation is no different from others in pathology in which, for example, Reed-Sternberg cell variants in an appropriate setting is diagnostic of recurrent Hodgkin's lymphoma, yet classic Reed-Sternberg cells are required for the initial diagnosis. Second, the prostate has been removed, such that the finding of a few atypical glands in soft tissue without surrounding benign prostate tissue, as seen in Figure 7, is not expected and indicates recurrent cancer. Although in $14 \%$ of cases we found benign prostate tissue postoperatively, these glands were histologically bland and typically away from the recurrent cancer. Consequently, the presence of a few atypical glands is often diagnostic of recurrent prostate cancer, although those same glands sampled on a needle biopsy of the intact prostate might be called suspicious but not diagnostic of cancer. Although in the current study we did not use immunohistochemistry for high-molecular-weight cytokeratin, this adjunct may be useful in difficult cases. Data from our studies and the literature demonstrate that one cannot rely on the clinical, radiologic, or radical prostatectomy data to establish a diagnosis of locally recurrent prostate cancer. The diagnosis of locally recurrent cancer must be based on a constellation of the histologic findings along with the history of surgery.

\section{REFERENCES}

1. Morton RA, Steiner MS, Walsh PC. Cancer control following anatomical radical prostatectomy: an interim report. J Urol 1991;145:1197-200.

2. Cadeddu JA, Partin AW, DeWeese TL, Walsh PC. Long-term results of radiation therapy for prostate cancer recurrence following radical prostatectomy. J Urol 1998;159:173-8.

3. Connolly JA, Shinohara K, Presti JC Jr, Carrol PR. Local recurrence after radical prostatectomy: characterization in size, location, and relationship to prostate-specific antigen and surgical margins. Urology 1986;41:225-31.

4. Fowler JE Jr, Brooks J, Pandey P, Seaver L. Variable histology of anastomotic biopsies with detectable prostate specific antigen after radical prostatectomy. J Urol 1995;153:1011-4.

5. Saleem MD, Sanders H, El Naser MA, El-Galley R. Factors predicting cancer detection in biopsy of the prostatic fossa after radical prostatectomy. Urology 1998;51:283-6.

6. Abi-Aad AS, Macfarlane MT, Stein A, DeKernion JB. Detection of local recurrence after radical prostatectomy by prostate specific antigen and transrectal ultrasound. J Urol 1992; $147: 952-5$.

7. Foster LS, Jajodia P, Fournier G Jr, Shinohara K, Carroll P, Narayan P. The value of prostate specific antigen and transrectal ultrasound guided biopsy in detecting prostatic fossa recurrences following prostatectomy. J Urol 1993;149:1024-8.

8. Lange PH, Lightner DJ, Medini E, Reddy PK, Vassella RL. The effect of radiation therapy after radical prostatectomy in patients with elevated prostate specific antigen levels. J Urol 1990;144:927-33.

9. Van Den Ouden D, Bentvelsen FM, Boevé ER, Schröder FH. Positive margins after radical prostatectomy: correlation with local recurrence and distant progression. Br J Urol 1993;72:489-94.

10. Steinberg DM, Sauvageot J, Piantadosi S, Epstein JI. Correlation of prostate needle biopsy and radical prostatectomy Gleason grade in academic and community settings. Am J Surg Pathol 1997;21:566-76. 